\title{
The Burden of Opioid-Induced Constipation: Discordance Between Patient and Health Care Provider Reports
}

\author{
Robert J. LoCasale, PhD, MS; Catherine Datto, MD, MS; Hilary Wilson, PhD; \\ Karen Yeomans, BSc; and Karin S. Coyne, PhD, MPH
}

\begin{abstract}
BACKGROUND: Opioid-induced constipation (OIC), a common side effect of opioid treatment for chronic pain, affects patient health-related quality of life (HRQL) and may prompt some patients to lower the dose or alter adherence to their opioid medication, compromising pain relief. Although health care providers (HCPs) are aware of the potential for OIC, patients may not inform their HCPs of their OIC symptoms, and HCPs may not initiate conversation regarding OIC if their patients' pain is controlled. Patients often try to address OIC symptoms on their own by using natural approaches or over-the-counter options. When OIC is discussed in an office visit, HCPs typically recommend conventional laxatives to relieve symptoms, but the efficacy of this approach is unproven and often suboptimal. In many areas of medicine, HCP perceptions of the impact of adverse effects of treatment on a patient's HRQL do not align with the patient's experience.

OBJECTIVES: To (a) describe HCP-reported understanding of his or her patients' experiences with OIC and (b) evaluate the level of agreement or discordance in perception between patients and their HCPs of OIC's impact on clinical outcomes.
\end{abstract}

METHODS: This was a prospective, longitudinal, observational cohort study conducted in the United States, Canada, Germany, and the United Kingdom (NCT01928953) in patients aged 18 to 85 years who had been receiving daily opioid therapy for $\geq 4$ weeks for chronic noncancer pain with presence of OIC in the past 2 weeks. Data were collected from retrospective chart reviews, HCP questionnaires, and web-based patient surveys. Eligible patients enrolled online and completed the Patient Assessment of Constipation-Symptoms, the Work Productivity and Activity Impairment Questionnaire-Specific Health Problem, the EuroQOL 5 Dimensions, and the Global Assessment of Treatment Benefit, Satisfaction, and Willingness to Continue standardized questionnaires. The patient-reported component included 1 baseline survey and 8 follow-up surveys over 24 weeks. HCPs completed a web-based survey at baseline and at week 24 to assess their perceptions of OIC burden, treatment patterns, laxative use, and overall treatment satisfaction. The correspondence of patient- and HCP-reported data was evaluated for all similar outcomes from these 2 databases.

RESULTS: Patients $(\mathrm{N}=489)$ reported a mean $(\mathrm{SD})$ number of bowel movements (BMs) per week and spontaneous BMs per week of 3.7 (2.9) and 1.4 (2.3), respectively, at baseline. Most (87\%) reported chronic pain of $\geq 2$ years duration; $65 \%$ had used opioids for $\geq 2$ years; and the mean pain score at baseline was 6.3 , consistent with a moderate-to-severe pain population. Most ( $97 \%$ ) patients at baseline reported any gastrointestinalrelated symptom of at least moderate intensity, with $82 \%$ reporting the same intensity of any symptom at week 24 . Of the 405 patients who had seen their HCPs in the past month, $63 \%$ reported that they had spoken to their HCPs about constipation, and 62\% reported that their HCPs had asked them if they had constipation in the past month. The proportion of agree- ment between HCPs and their patients on the presence of constipation at baseline was $61 \%$. Similar average pain ratings between HCPs and patients (5.9 vs. 6.0) at week 24 suggested clear communication regarding the level of pain experienced by the patient; however, OIC symptoms, laxative use and effectiveness, and impact of OIC on pain management and HRQL were not fully appreciated by HCPs.

CONCLUSIONS: The importance and severity of OIC are perceived differently by patients and their HCPs, a discordance that complicates pain management and demonstrates a need for greater communication. These disparate perceptions indicate a need for clinical education and coordination of care by HCPs to improve understanding and proactively manage OIC in patients with chronic noncancer pain.

J Manag Care Spec Pharm. 2016;22(3):236-45

Copyright $\odot 2016$, Academy of Managed Care Pharmacy. All rights reserved.

\section{What is already known about this subject}

In the United States, patient surveys and medical record reviews suggest that $17 \%-67 \%$ of patients experience opioid-induced constipation (OIC) as an adverse event of their opioid pain medication. In the majority of these patients, the symptoms of OIC affect activities of daily living and health-related quality of life (HRQL) and prompt patients to decrease the dose or temporarily stop using their opioid medication to relieve their constipation.

Discordance between the perceptions of health care providers (HCPs) and patients is found in many areas of medicine and may compromise patient care and disease management.

\section{What this study adds}

This study investigated the perceptions of patients and HCPs about the burden of OIC with regard to symptoms, adequacy of pain management, use and effectiveness of laxative therapy, and activities of daily living in patients receiving opioids for chronic noncancer pain with the presence of OIC.

Similar average pain ratings between HCPs and patients (5.9 vs. 6.0) at week 24 suggested clear communication regarding the level of pain experienced by the patient.

However, OIC symptoms, laxative use and effectiveness, and the negative effects of OIC on pain management and HRQL were not fully appreciated by HCPs. 
$\mathrm{E}$ ffective communication between patients and their health care providers (HCPs) is recognized as essential for optimal patient outcomes, but it is often difficult to achieve in today's time-constrained health care environment. ${ }^{1-3}$ Discordance between the perceptions of HCPs and patients is found in many areas of medicine and may compromise patient care and disease management. For example, in surveys carried out to determine the impact of gastroesophageal reflux disease (GERD) on health-related quality of life (HRQL), a disconnect was noted between patients and HCPs regarding treatment of GERD, with HCPs underestimating the use of over-the-counter therapies by their patients with GERD. ${ }^{4}$ Similarly, results obtained on HRQL questionnaires completed by HCPs and their patients with melanoma who were receiving adjuvant PEGylated interferon- $a 2 b$ therapy showed that HCPs significantly underestimated the HRQL impairments that their patients were experiencing. ${ }^{5}$

Opioids are commonly used to treat chronic noncancer pain. ${ }^{6}$ Data from U.S. patient surveys and medical record reviews suggest that $17 \%-67 \%$ of patients experience constipation as an adverse effect of their opioid pain medication. ${ }^{7-9}$ The absence of general agreement on the magnitude of the problem is an indication that patients may have different perceptions of when opioid-induced constipation (OIC) becomes an issue for them. This lack of agreement further underscores the importance of better understanding patient-physician communication. Patients with OIC may experience abdominal discomfort, nausea, gas, decreased appetite, reflux, bloating, straining, incomplete evacuation, and hard bowel movements (BMs). ${ }^{10,11}$ In the majority ( $\geq 80 \%$ ) of patients, these symptoms affect activities of daily living and HRQL, ${ }^{12}$ with individuals receiving opioids who developed OIC reporting significantly worse HRQL than those who did not develop OIC. ${ }^{13}$

Nonpharmacologic interventions, such as increasing fluid and dietary intake of fiber, are common first-line approaches for treating OIC but are rarely sufficient to manage the condition. ${ }^{11}$ Laxatives are often used to treat OIC, but they have limited efficacy. ${ }^{14,15}$ Laxatives exist in a variety of formulations, including surfactants that soften the stool, osmotic agents that retain water in the gastrointestinal (GI) tract, and stimulants that promote motor activity and the secretion of fluids in the GI tract. ${ }^{15,16}$ Bulk-forming laxatives may not be effective for the treatment of OIC because the receptors stimulated by the increased bulk are blocked by opioids. ${ }^{15}$ Safety and low cost render over-the-counter laxatives and natural remedies appropriate first choices for pharmacotherapy; however, they have no ability to block opioid actions at GI opioid receptors. ${ }^{15}$

The symptoms of OIC may prompt patients to decrease the dose or stop using their opioid medication to relieve their constipation. $^{12,14}$ In the international, Internet-based Patient Reports of Opioid-Related Bothersome Effects (PROBE 1) survey, $33 \%$ of patients reported that they had skipped, decreased, or ceased use of opioids to facilitate having a BM. ${ }^{12}$ Thus, the inability to achieve a balance between pain relief and development of constipation impairs HRQL and compromises effective pain management. ${ }^{17} \mathrm{~A}$ better understanding of OIC or more active HCP participation in monitoring and managing OIC may help with this balance.

The objectives of this study were (a) to describe HCPreported understanding of his or her patients' experiences with OIC, including the symptoms experienced, adequacy of treatment, and adequacy of pain management among patients receiving chronic opioid therapy for chronic noncancer pain; and (b) to evaluate the level of agreement or discordance in perception between patients and their HCPs of OIC's impact on clinical outcomes.

\section{Methods}

\section{Study Design}

This was a prospective, longitudinal, observational cohort study conducted in the United States, Canada, Germany, and the United Kingdom (NCT01928953). A detailed description of the methods of this study has been published. ${ }^{17}$ Briefly, patients with usual-care visits to primary care, pain, or other specialty clinics (neurology, orthopedics, rheumatology) were recruited. Data were collected from web-based patient surveys, retrospective chart reviews, and HCP questionnaires. Both patients and their respective HCPs completed surveys, and the responses on the HCP survey were based on HCP knowledge of his or her patients who completed the patient survey. The protocol and informed consent forms were reviewed and approved by institutional review boards and ethics committees to meet country- and site-specific requirements.

\section{Patients}

Patients aged 18 to 85 years who had been receiving daily opioid therapy for $\geq 4$ weeks to treat chronic noncancer pain with presence of $\mathrm{OIC}$ in the 2 weeks before screening were eligible for enrollment. Patients were receiving a minimum total daily dose of $30 \mathrm{mg}$ oral morphine or equianalgesic amounts of 1 or more other opioid therapies and were expected to continue on opioid therapy for $\geq 6$ months. Presence of OIC was based on patient-reported symptoms. Patients were required to report laxative use if the number of BMs in the past 2 weeks was $\geq 3$ per week, whereas those with $<3$ BMs per week in the past 2 weeks were eligible regardless of reported laxative use. Patients who did not report laxative use were required to report at least 1 symptom of OIC (e.g., straining during BM, hard/lumpy stools, or sensation of incomplete emptying) in the past 2 weeks. Patients who were unable or who refused to provide informed consent, who were unable to use or were without access to a computer with Internet connectivity to complete the web-based survey, or who had a history of chronic constipation were excluded. 


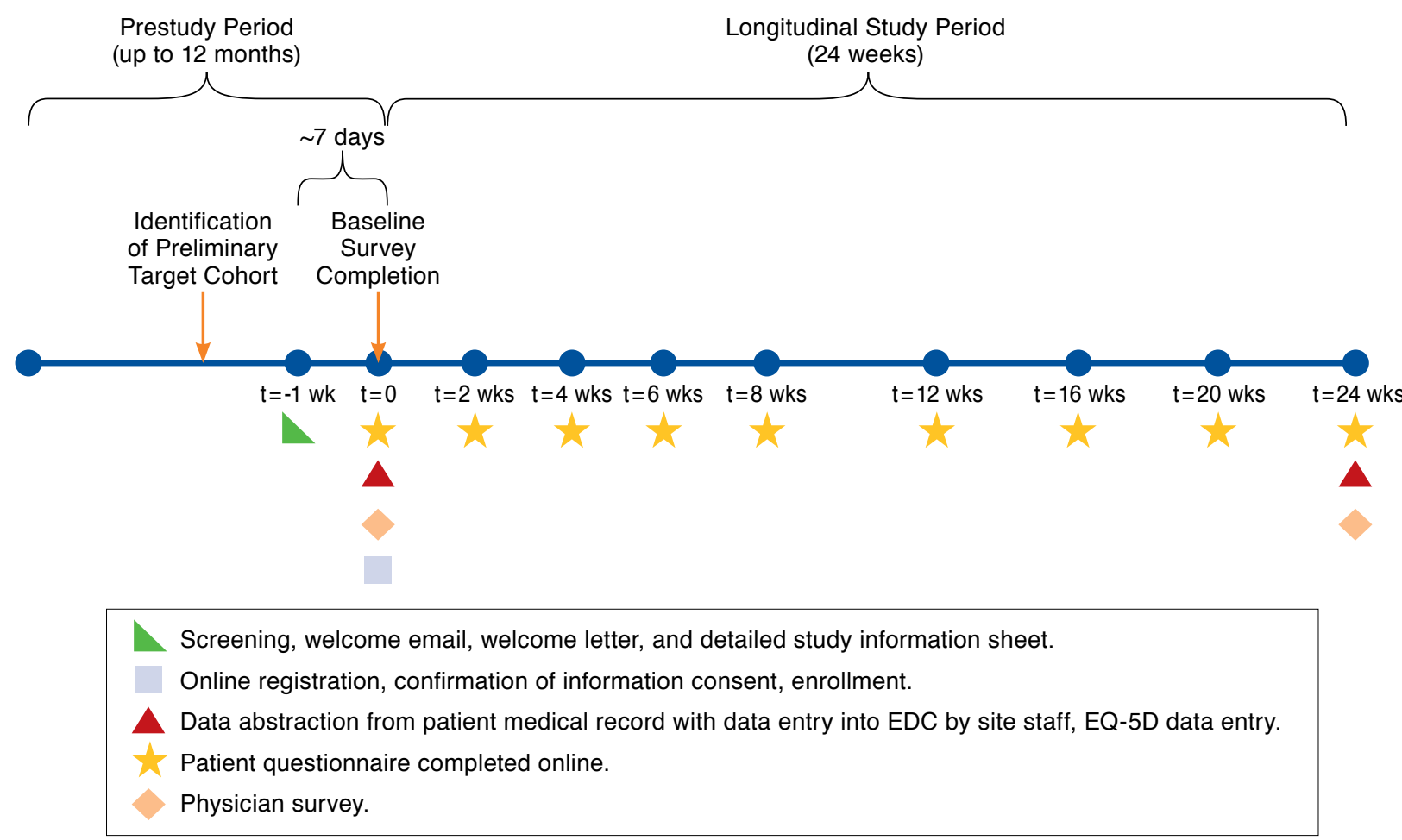

EDC=electronic data capture; $E Q-5 D=$ EuroQOL 5 Dimensions; $t=$ time; whs $=$ weeks.

\section{Assessments}

Eligible patients enrolled online and completed web-based surveys to provide data regarding sociodemographic and clinical characteristics, treatment patterns, BMs, constipation history, laxative use, and patient-reported outcome measures, including the Patient Assessment of Constipation-Symptoms (PACSYM $),{ }^{18}$ with 5 additional symptom items to assess nausea, vomiting, flatulence, GERD, and headache/migraine. Other standardized questionnaires in the patient survey included the Work Productivity and Activity Impairment QuestionnaireSpecific Health Problem, ${ }^{19}$ the EuroQOL 5 Dimensions,${ }^{20}$ and the Global Assessment of Treatment Benefit, Satisfaction, and Willingness to Continue. ${ }^{21}$ The prospective, patient-reported component contained 1 baseline survey and 8 follow-up surveys over 24 weeks. Patients were nominally remunerated for their time to complete each survey. Retrospective chart reviews assessed patient medical history, pharmacotherapy (including opioid and laxative prescriptions), and health care resource utilization at 2 points in time-at baseline, which included the 12 months leading up to the baseline visit, and at the end of the 6-month prospective study period (week 24 visit), covering a total period of 18 months (Figure 1). HCPs completed an
Internet-based questionnaire to assess their perception of OIC burden, symptoms, and treatment patterns for OIC, laxative use, and overall OIC treatment satisfaction of their patients at baseline and at week 24 for each patient who completed a baseline web-based survey.

\section{Data Analysis}

All analyses were performed with SAS software package 9.4 (SAS Institute, Cary, NC). Owing to the extensive nature of the objectives and number of analyses, descriptive statistics were applied. Summary measures such as means and standard deviations (SDs) for continuous variables and counts, frequencies, and percentages for categorical variables were reported; no statistical significance tests were applied. The correspondence of patient- and HCP-reported data was evaluated for all similar outcomes from these 2 databases, including OIC symptoms and treatment use. All data were analyzed without imputation for missing responses.

\section{Results}

\section{Survey Metrics}

Of the 617 patients initially recruited, 500 (81\%) were enrolled and completed the patient baseline survey. Of those, 489 (98\%) 
TABLE 1 Demographic and Clinical Characteristics of the Overall Patient Population

\begin{tabular}{|c|c|c|c|c|c|}
\hline \multirow[b]{2}{*}{ Male, \% (n) } & \multicolumn{2}{|c|}{ Patients $(\mathrm{N}=489)$} & & Patien & $=489)$ \\
\hline & 37.8 & $(185)$ & \multicolumn{3}{|l|}{ Employment status, \% (n) } \\
\hline Age, years, mean [SD] & 52.6 & [11.6] & Employed, full-time & 19.0 & (93) \\
\hline \multicolumn{3}{|l|}{ Race, \% (n) } & Employed, part-time & 8.0 & (39) \\
\hline White & 84.9 & $(415)$ & Studying full- or part-time & 1.2 & (6) \\
\hline Black & 4.9 & $(24)$ & Both employed and studying & 0.6 & (3) \\
\hline Asian & 4.5 & $(22)$ & Unable to work because of disability or handicap & 34.2 & $(167)$ \\
\hline Hispanic/Latino/Latin American & 1.8 & (9) & Unemployed & 4.5 & $(22)$ \\
\hline Other & 2.9 & (14) & Not currently working (e.g., full-time homemaker) & 3.7 & (18) \\
\hline Prefer not to state & 0.8 & (4) & Retired & 21.1 & $(103)$ \\
\hline Missing & 0.2 & $(1)$ & Other & 1.4 & $(7)$ \\
\hline \multicolumn{3}{|l|}{ Marital status, \% (n) } & Prefer not to answer & 6.1 & (30) \\
\hline Single, never married & 14.7 & $(72)$ & Missing & 0.2 & (1) \\
\hline Married & 52.4 & $(256)$ & Body mass index, $\mathrm{kg} / \mathrm{m}^{2}$, mean [SD] & 29.7 & [7.3] \\
\hline Living together, not married & 5.3 & $(26)$ & \multicolumn{3}{|l|}{ Health rating, \% (n) } \\
\hline Common-law partnership & 2.2 & $(11)$ & Excellent & 1.4 & (7) \\
\hline Divorced & 12.9 & (63) & Very good & 6.7 & (33) \\
\hline Separated & 1.4 & $(7)$ & Good & 29.7 & $(145)$ \\
\hline Widowed & 4.5 & $(22)$ & Fair & 42.3 & $(207)$ \\
\hline Prefer not to answer & 5.3 & $(26)$ & Poor & 19.2 & (94) \\
\hline Missing & 1.2 & (6) & Missing & 0.6 & (3) \\
\hline \multicolumn{3}{|l|}{ Type of chronic pain, $\%(n)^{a}$} & Duration of chronic pain, years, mean [SD] & 9.8 & [8.9] \\
\hline Back pain & 76.7 & $(375)$ & $<2$ years, $\%(n)$ & 13.3 & $(65)$ \\
\hline Joint pain & 51.5 & $(252)$ & $\geq 2$ years, $\%(n)$ & 86.7 & $(424)$ \\
\hline Fibromyalgia & 14.5 & $(71)$ & Duration of opioid medication, years, mean [SD] & 6.4 & [6.3] \\
\hline Headache or migraine & 16.0 & $(78)$ & $<2$ years, $\%(\mathrm{n})$ & 23.9 & $(117)$ \\
\hline Osteoarthritis & 19.4 & (95) & $\geq 2$ years, $\%(n)$ & 65.4 & $(320)$ \\
\hline Rheumatoid arthritis & 8.4 & $(41)$ & Unknown, \% (n)b & 10.6 & $(52)$ \\
\hline Neuralgia & 23.5 & $(115)$ & Pain severity rating & & \\
\hline Pain syndrome & 33.1 & $(162)$ & Average pain in last 24 hours, mean [SD] & 6.3 & [1.8] \\
\hline Other & 15.5 & $(76)$ & Average pain in last 7 days, mean [SD] & 6.3 & [1.7] \\
\hline
\end{tabular}

met criteria confirming the presence of OIC. Chart reviews were completed for 486 of 489 (99\%) eligible patients at baseline and for 477 (98\%) patients at week 24. HCP baseline and week 24 surveys were completed for 464 (95\%) and 457 (94\%) patients, respectively.

Patient Demographic and Clinical Characteristics and Baseline Constipation History. Demographic and clinical characteristics of the overall patient population are shown in Table 1. Most patients were female (62\%) and white (85\%), with a mean age of 53 years. More than half of the patients (62\%) rated their overall health as fair or poor. Twenty-seven percent of patients were working full- or part-time, and 34\% were unable to work because of disability. The most frequently reported pain conditions were back pain (77\%) and joint pain (52\%). The mean (SD) duration of chronic pain and the mean (SD) duration of chronic opioid use was 9.8 (8.9) years and 6.4
(6.3) years, respectively. Most (87\%) patients reported chronic pain of $\geq 2$ years in duration, and $65 \%$ had used opioids for $\geq 2$ years. The mean pain score at baseline was 6.3 , both for pain in the last 24 hours and for pain in the last 7 days, consistent with a moderate-to-severe pain population.

The mean (SD) number of BMs per week was 3.7 (2.9), and the mean (SD) number of spontaneous BMs per week was 1.4 (2.3) (Table 2). On the basis of PAC-SYM and additional symptom assessments, $97 \%$ of patients at baseline reported any symptom of at least moderate intensity that remained high throughout follow-up, with $82 \%$ reporting the same intensity of any symptom at week 24 . Laxative use was sufficient (defined as use of $\geq 1$ laxative $\geq 4$ times over the past 2 weeks) in $48 \%$, insufficient in $25 \%$, and absent in $27 \%$ of patients. Of the 405 patients who had seen their HCPs in the past month, $63 \%$ reported that they had spoken to their HCPs about 


\begin{tabular}{|c|c|c|}
\hline \multicolumn{3}{|c|}{$\begin{array}{l}\text { Patient-Reported Constipation History } \\
\text { and Opioid Use Management at } \\
\text { Baseline }\end{array}$} \\
\hline & & $\begin{array}{c}\text { Overall } \\
(\mathrm{N}=489)\end{array}$ \\
\hline $\begin{array}{l}\text { Over the past } 2 \text { weeks, how many BMs } \\
\text { were spontaneous? }\end{array}$ & & $\mathrm{N}=488$ \\
\hline Mean [SD] & 1.4 & {$[2.3]$} \\
\hline \multicolumn{3}{|c|}{ Ideally, how often would you like to have BMs? \% (n) } \\
\hline More than once a day & 12.7 & $(62)$ \\
\hline Once a day & 69.9 & $(342)$ \\
\hline Every other day & 10.4 & $(51)$ \\
\hline 2-3 times per week & 5.9 & $(29)$ \\
\hline Once per week & 1.0 & $(5)$ \\
\hline $\begin{array}{l}\text { If you saw your HCP in the last month, } \\
\text { did you speak about constipation? }\end{array}$ & & $\mathrm{N}=405$ \\
\hline Yes, \% (n) & 63.2 & $(256)$ \\
\hline $\begin{array}{l}\text { If you saw your HCP in the last month, did } \\
\text { your HCP ask if you were having constipation } \\
\text { in the last month? }\end{array}$ & & $\mathrm{N}=405$ \\
\hline Yes, \% (n) & 61.8 & $(251)$ \\
\hline $\begin{array}{l}\text { If you did not speak to your HCP about } \\
\text { constipation, why not? \% (n) }\end{array}$ & & $N=149$ \\
\hline Constipation not a problem & 4.0 & (6) \\
\hline Embarrassed & 9.4 & (14) \\
\hline Ran out of time & 4.7 & $(7)$ \\
\hline $\begin{array}{l}\text { Concerned about need to change/reduce pain } \\
\text { medication }\end{array}$ & 13.4 & (20) \\
\hline Discussed with doctor in the past & 58.4 & $(87)$ \\
\hline Other & 10.1 & $(15)$ \\
\hline \multicolumn{3}{|c|}{$\begin{array}{l}\text { Since starting opioid medication, have you been experiencing } \\
\text { constipation or worsening of current constipation? \% (n) }\end{array}$} \\
\hline Yes & 93.7 & $(458)$ \\
\hline Missing & 0.2 & $(1)$ \\
\hline $\begin{array}{l}\text { How long after did you begin experiencing } \\
\text { constipation? } \%(\mathrm{n})\end{array}$ & & $\mathrm{N}=458$ \\
\hline 1 day after beginning & 4.4 & $(20)$ \\
\hline Within first week & 34.7 & $(159)$ \\
\hline 2-4 weeks & 26.0 & (119) \\
\hline 5-8 weeks & 6.1 & $(28)$ \\
\hline$>8$ weeks & 5.2 & $(24)$ \\
\hline Don't remember & 23.6 & $(108)$ \\
\hline \multicolumn{3}{|c|}{$\begin{array}{l}\text { In past } 7 \text { days, did you change how you used your opioid } \\
\text { medication(s) so that you could have BMs? \% yes (n)a }\end{array}$} \\
\hline If yes, how change, ${ }^{b} \mathrm{n}(\%)$ & 7.6 & $(37)$ \\
\hline No longer take my pain medication & 2.7 & (1) \\
\hline Reduced how much of my pain medication I use & 43.2 & (16) \\
\hline $\begin{array}{l}\text { Temporarily interrupted the use of pain } \\
\text { medication }\end{array}$ & 48.6 & (18) \\
\hline Switched to a different pain medication & 10.8 & (4) \\
\hline Other & 2.7 & $(1)$ \\
\hline \multicolumn{3}{|c|}{$\begin{array}{l}\text { a8\% of responders. } \\
\text { bParticipants were able to report more than } 1 \text { response. } \\
B M=\text { bowel movement; } H C P=\text { health care provider; } S D=\text { standard deviation. }\end{array}$} \\
\hline
\end{tabular}

constipation, and $62 \%$ reported that their HCPs had asked them if they had constipation in the past month. Results did not vary appreciably across countries (data not shown),
TABLE 3 HCP-Reported Awareness of Patients' Bowel Symptoms and OIC at Baseline

\begin{tabular}{|c|c|c|}
\hline & & $\begin{array}{c}\text { Overall } \\
(\mathrm{N}=464)^{\mathrm{a}}\end{array}$ \\
\hline Knows patient is experiencing OIC, \% (n) & 65.1 & $(302)$ \\
\hline \multicolumn{3}{|l|}{ Patient discussed OIC symptoms/concerns, \% (n) } \\
\hline Yes & 76.9 & (357) \\
\hline No & 22.8 & $(106)$ \\
\hline Missing & 0.2 & (1) \\
\hline Patient's pain level & & $\mathrm{N}=404$ \\
\hline Mean [SD] & 6.3 & [1.8] \\
\hline $\begin{array}{l}\text { Patient complained about having too few } \\
\text { BMs per week, \% (n) }\end{array}$ & 58.4 & $(271)$ \\
\hline $\begin{array}{l}\text { Typical number of BMs this patient has per } \\
\text { week }\end{array}$ & & $\mathrm{N}=193$ \\
\hline Mean [SD] & 2.5 & [1.4] \\
\hline Don't know, \% (n) & 16.8 & $(78)$ \\
\hline $\begin{array}{l}\text { Number of BMs per week this patient considers } \\
\text { to be too few }\end{array}$ & & $\mathrm{N}=90$ \\
\hline Mean $[S D]$ & 3.5 & {$[2.1]$} \\
\hline Don't know, \% (n) & 39.0 & $(181)$ \\
\hline \multicolumn{3}{|c|}{ How does OIC affect this patient's quality of life? \% (n) } \\
\hline Life can be carried on as usual & 39.7 & $(184)$ \\
\hline Quality of life is restricted & 34.1 & $(158)$ \\
\hline Lifestyle is highly affected & 6.5 & $(30)$ \\
\hline Don't know & 19.2 & (89) \\
\hline Missing & 0.6 & (3) \\
\hline
\end{tabular}

although fewer patients in the United Kingdom discussed their OIC with their HCPs (46\%) as opposed to patients in other countries (United States, 64\%; Canada, 52\%; Germany, 76\%). Of the 149 participants who did not speak to their HCPs about constipation in the past month, more than half (58\%) had not done so because they had spoken to their HCPs about constipation in the past, whereas 13\% had not done so because of concerns that their pain medication would be changed or the dose would be reduced. In addition, $8 \%$ of patients reported that they adjusted their opioid pain medication to have a BM, with the majority experiencing a worsening of pain as a result. Of these patients, $49 \%$ temporarily interrupted and $43 \%$ reduced the use of their pain medication.

HCP Awareness of Patients' OIC and Impact of OIC on Patients' Lives: Baseline Results. Overall, $77 \%$ of HCPs reported that they discussed OIC symptoms and concerns with patients; however, only $65 \%$ were actually aware that the patients met criteria for OIC and only 58\% of HCPs reported that patients complained of having too few BMs per week (Table 3). A subset of HCPs responded to additional follow-up questions about the specific number of BMs patients experienced per week and what number of BMs per week a patient 
FIGURE 2 Agreement of Patient- and HCP-Reported OIC Symptoms at Baseline: Overall

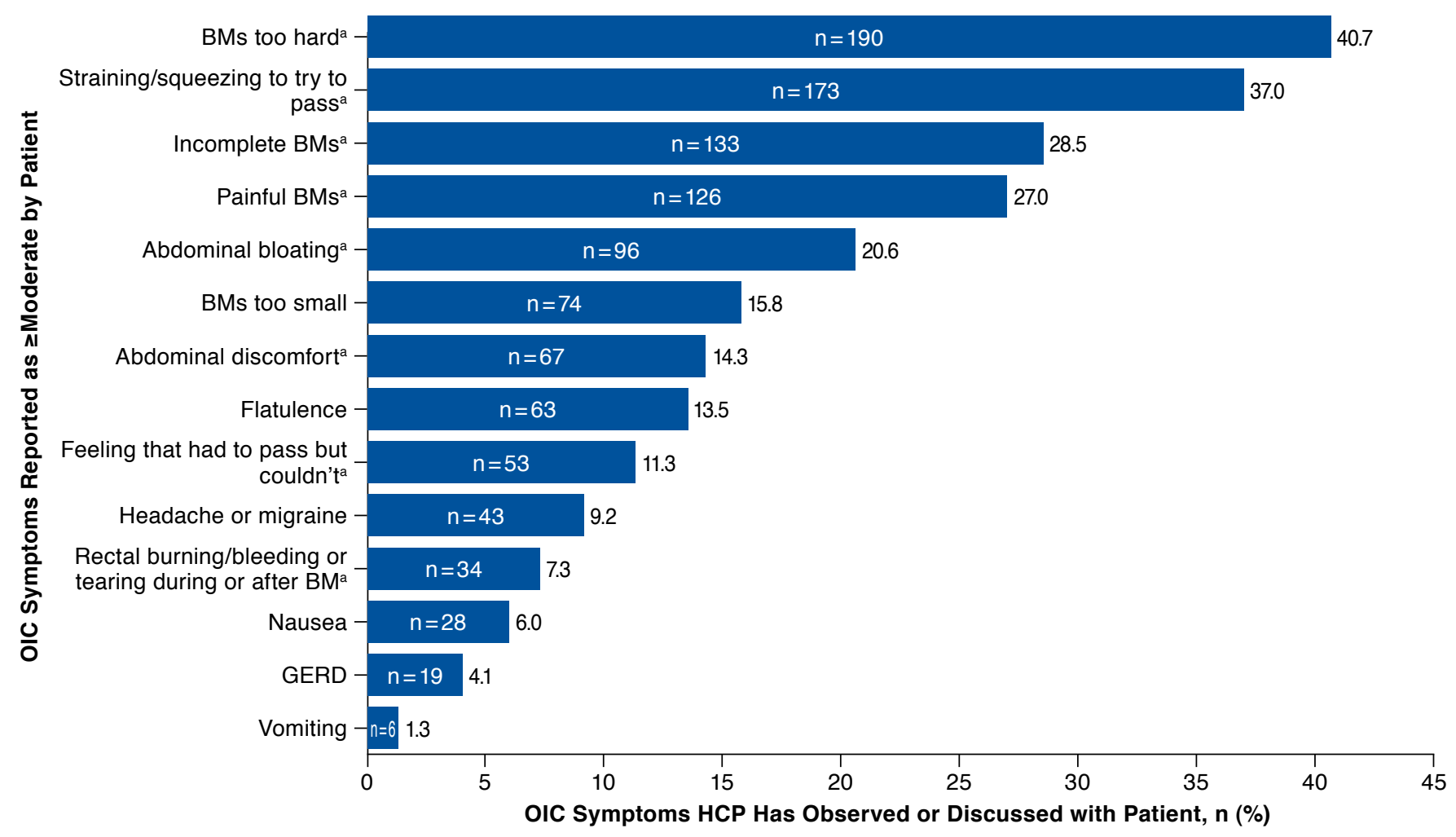

aFrom PAC-SYM.

$B M=$ bowel movement; GERD = gastroesophageal reflux disease; HCP=health care provider; OIC=opioid-induced constipation; PAC-SYM=Patient Assessment of Constipation-Symptoms.

considered too few. Of those responding, HCPs reported that their patients experienced 2.5 BMs per week on average, which is $1 \mathrm{BM}$ per week fewer, on average, than what patients actually reported. Additionally, $17 \%$ of HCPs were unaware of the number of BMs a patient experienced per week, and 39\% were unaware of how many BMs were considered too few, consistent with the reported lack of awareness by HCPs that patients met criteria for OIC. Of those who did provide a response, HCPs reported that 3.5 BMs per week on average were considered too few for patients, which is still under the desired $1 \mathrm{BM}$ or more per day that $83 \%$ of patients reported would be ideal. Almost half $(48 \%)$ of HCPs responded that treatments to relieve OIC were moderately to completely adequate. Forty percent of HCPs reported that they believed that OIC did not have an effect on patient quality of life with regard to activities of daily living.

Concordance Between Patient- and HCP-Reported OIC and Pain Symptoms at Baseline. The proportion of agreement between HCPs and patients on the presence of constipation at baseline was $61 \%$. The most commonly reported symptoms rated as moderate to severe in intensity by patients were straining/ squeezing to pass a BM (83\%), BMs too hard (75\%), flatulence (69\%), incomplete BM (69\%), bloating in abdomen (69\%), painful BM (67\%), and abdominal discomfort (64\%); however, the proportion of HCPs who had observed or discussed each OIC symptom reported by the patient as being of moderate or greater severity ranged from $1 \%$ to $41 \%$ (Figure 2).

In contrast with OIC symptomology, HCPs were well aware of patients' pain severity, with both patients and HCPs reporting an average pain rating of 6.3. Nevertheless, HCPs overestimated how well patients managed their pain and OIC concurrently. Although $49 \%$ of patients reported that OIC interfered moderately or completely with the ability of their opioid medication to adequately control their pain, a majority (73\%) of HCPs reported that their patients' pain was mostly or adequately managed (Figure 3). This pattern continued at week 24 , with 46 participants (45\%) reporting moderate to complete interference of OIC with their pain management, and $73 \%$ of HCPs reporting their patients' pain was mostly or adequately managed. The proportion of agreement between 
FIGURE 3 Discordance Between Patient and HCP Reports of OIC Interference with Adequate Pain Management ${ }^{a}$

\section{A.Patient Report: How Much Does Constipation Interfere with Ability to Control Pain}
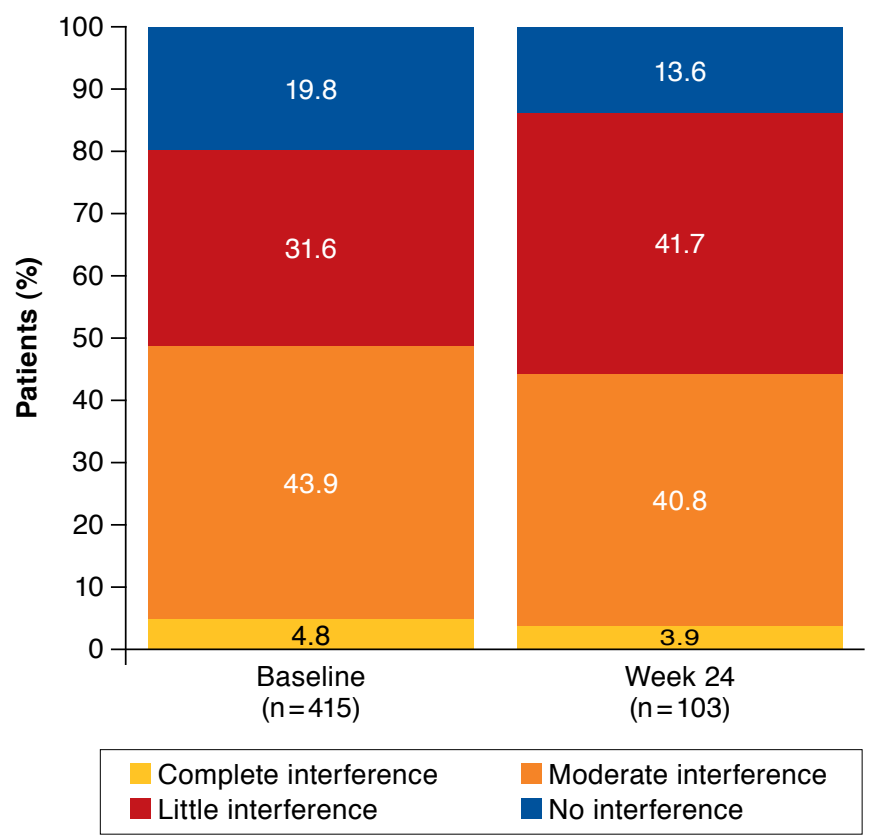

B. HCP Report: How Much Does OIC Interfere with This Patient's Adequate Pain Management

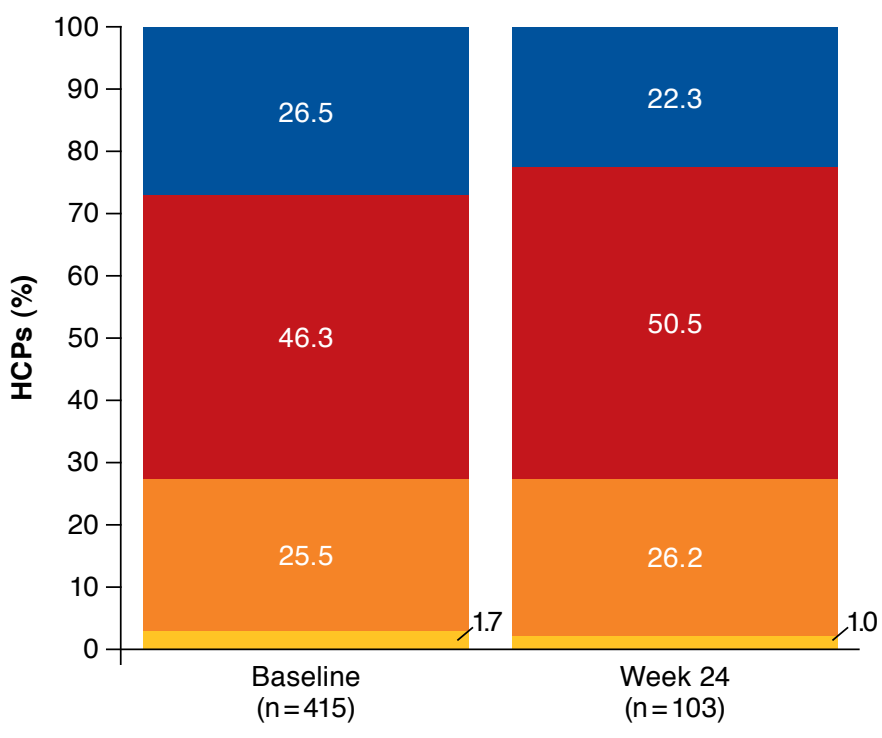

Not at all managed
Mostly managed
Moderately managed - Adequately managed

a Survey item was only completed for week 24 if HCP reported an OIC discussion on symptoms or concerns within the past 6 months.

$\mathrm{HCP}=$ health care provider; OIC=opioid-induced constipation.

HCPs and patients on the current use of laxatives was 55\%, and the proportion of agreement on the level of benefit and satisfaction from laxatives varied between patients and HCPs. Among the 305 patients who reported little to no benefit of laxative treatment at baseline, 53\% of their HCPs reported moderate-to-complete satisfaction with laxative treatment at baseline. A similar pattern was present at week 24 , with $60 \%$ of HCPs overestimating treatment benefit. For satisfaction, 99 patients (51\%) reported they were a little to very dissatisfied with laxative treatment at baseline, with 54\% of HCPs overestimating the baseline level of satisfaction of their patients as moderate-to-complete satisfaction. A similar pattern was observed at week 24 , when $62 \%$ of HCPs overestimated their patients' satisfaction.

Results at Week 24 Follow-up. Overall, 72\% ( $=330)$ of HCPs reported that they had a follow-up visit or other consultation with their patients since the baseline visit. The patient-reported mean pain score was similar to that estimated by HCPs (5.9 vs. 6.0) at week 24 for pain in the last 24 hours and for pain in the last 7 days. Of the 330 HCPs who had a follow-up visit, $38 \%(n=125)$ reported having discussed OIC symptoms and concerns with patients. Of these, HCPs responded that $75 \%$ of patients had taken laxatives to manage their OIC since the baseline survey and that about half (52\%) had achieved moderate-to-complete satisfaction with laxative treatment. Almost half $(46 \%)$ of the HCPs responded that treatments to relieve OIC were moderately to completely adequate. About half (54\%) of HCPs expressed that OIC had little to no interference with the patients' reports of HRQL. Of those visits in which the patients discussed OIC symptoms/concerns $(n=125)$, the most common treatment recommendations were to decrease the frequency of pain medication ( $39 \%$ of HCPs, $n=49$ ) or to take an opioid holiday ( $47 \%$ of HCPs, $n=59$ ).

\section{Discussion}

In this study, HCP-reported understanding of the patient experience with OIC and pain management when receiving chronic opioid therapy for chronic noncancer pain was assessed. Analyses of consistency between patient and HCP responses on web-based surveys were interpreted as evidence of limited or incomplete HCP awareness of the patient disease burden of OIC. Identical average pain ratings by HCPs and patients at baseline and similar average pain ratings by HCPs and patients at week 24 indicated that there appears to be clear 
communication between HCPs and patients regarding the level of pain experienced by the patients; however, OIC symptoms (at baseline), laxative use, satisfaction and benefit, and the impact of OIC on the impairment of pain management are not fully appreciated by HCPs. A potential factor playing into this disparity is a lack of communication about OIC between HCPs and patients that may contribute to a lack of awareness of the impact of OIC by HCPs and ultimately patient dissatisfaction. However, identification of reasons for this disconnect would be a topic for further health promotion research.

Although most HCPs reported that they discussed OIC symptoms and pain severity with their patients, many were unaware of their patients' OIC symptom severity, level of laxative use, and limited benefits with laxative use, which increased somewhat during the 6 months of the study. Despite inclusion in a study of OIC, there appears to be very little advancement in the conversation between patients and HCPs and very little improvement in HCP insight into the disease burden of their patients. Thus, patients' ability to concurrently manage their pain and OIC is commonly underestimated by HCPs.

The reasons for a lack of discussion of OIC symptoms may be related to both HCPs and patients. The HCPs may run out of time or believe the patients should initiate the conversation, and/or the patients may not mention OIC symptoms for fear of losing the opioid medication. There may be a lack of appreciation of the relationship between OIC and opioid use, a belief that OIC is a condition that should be self-managed and not shared with a physician, or embarrassment.

Patients in this survey experienced chronic pain for an average of 9 years and used opioids for an average of 6 years. The gap in communication may have widened after being in this state for so long. A more proactive approach to prophylaxis, in which patients with chronic pain are targeted to have a conversation with their HCPs about constipation at the initiation of opioid therapy, may be a way to close this gap. It is also possible that the availability of better prescription therapies for OIC may also bring patients closer to their HCPs.

A previously published analysis of baseline data from this study showed that OIC had a negative impact on patient activities of daily living, work productivity, overall HRQL, and, for some patients, ability to manage pain. ${ }^{17}$ Other studies have also shown that OIC causes patients to compromise their pain management to avoid constipation..$^{13}$ In the current analysis, OIC interfered with patients' pain management more than HCPs realized.

Discordance in patient-physician communication is not restricted to patients with chronic pain and OIC. For example, patient overreporting of symptom severity and underrating of HRQL compared with physician assessments has been observed in surveys of patients with cardiovascular disease, psoriatic arthritis, rheumatic diseases, and systemic sclerosis. ${ }^{22-25}$ In a longitudinal study in cancer patients, those with severe constipation scores were more likely than patients with mild or moderate constipation scores to initiate a discussion of their symptoms with a physician at first consultation. ${ }^{26}$ Studies in other indications point to a failure of physicians to initiate a conversation about disease symptoms and quality of life, race/ethnicity-related discordance between patients and physicians, and the presence of comorbid conditions ${ }^{24}$ (e.g., physical inactivity, depression, fatigue, GI symptoms) as possible predictors of discordance between patient-physician perceptions. ${ }^{23,25-28}$ However, further research is needed to better understand the reasons for the discordance between patientphysician perceptions in patients with OIC.

We hypothesized that differences may exist between patient and HCP perceptions of the burden of OIC on the symptom experience, the adequacy of pain management, the use and effectiveness of laxative therapy, and the day-to-day activities of patients receiving opioids for chronic noncancer pain..$^{29}$

To improve patient care, the HCP treatment team may bridge this gap by including an assessment of OIC as part of routine appointments with patients receiving chronic opioid medication. Increasingly, patients are requested to complete brief questionnaires about their pain severity and other symptoms as part of the check-in process for routine-care visits. Inclusion of a single question to assess the presence of OIC may prompt physicians to follow up with patients about this issue. Clear treatment guidelines should be developed to establish best practices in the treatment of OIC when identified. A stepwise approach, with over-the-counter osmotic agents, surfactants, and/or stimulants as first-line therapy and prescription treatment options for those who do not respond to these therapies, should be considered. Clinical education and coordination of care by additional HCPs, including nursing staff, may add to the critical need to appreciate and proactively address the burden of disease. Development of a consensus definition of OIC and perhaps a validated assessment tool (i.e., patient-reported outcome) in OIC and empirically based treatment guidelines are areas of further research that could close the gap. Chronic pain management and treatment side effects, including OIC, present complex challenges for patients and their providers.

\section{Limitations}

This study was subject to limitations, given that it involved patient self-reports and may have been influenced by cultural factors, differences in clinical practice, and, in some cases, small sample size.

\section{Conclusions}

The importance and severity of OIC are perceived differently by patients and their HCPs, a discordance that complicates pain management and demonstrates a need for greater 
communication. These disparate perceptions indicate a need for clinical education and coordination of care by HCPs to improve understanding and proactively manage OIC in patients with chronic noncancer pain.

\section{Authors}

ROBERT J. LOCASALE, PhD, MS, is Group Director of Quality, Design \& Analytics, Global Medicines Development, Payer \& Real World Evidence, AstraZeneca Pharmaceuticals, Gaithersburg, Maryland; CATHERINE DATTO, MD, MS, is U.S. Medical Affairs Medical Lead, AstraZeneca Pharmaceuticals, Wilmington, Delaware; and KAREN YEOMANS, BSC, is Senior Manager and Senior Research Scientist, United BioSource Corporation, Montreal, $2 C$, Canada. HILARY WILSON, PhD, is Research Scientist, and KARIN S. COYNE, PhD, MPH, is Vice President, Research, Evidera, Bethesda, Maryland.

AUTHOR CORRESPONDENCE: Robert J. LoCasale, PhD, MS, Global Medicines Development, Payer \& Real World Evidence, AstraZeneca Pharmaceuticals, One MedImmune Way, 101ORD-2104A, Gaithersburg, MD 20878. Tel.: 301.398.0295; Fax: 302.885.9819; E-mail: robert.locasale@astrazeneca.com.

\section{DISCLOSURES}

This study was supported by AstraZeneca Pharmaceuticals. Datto and LoCasale are employees of AstraZeneca Pharmaceuticals. Wilson and Coyne are employees of Evidera and were paid scientific consultants to AstraZeneca in connection with this study. Yeomans is an employee of United BioSource Corporation, an Express Scripts Company, and was a paid scientific consultant to AstraZeneca in connection with this study and manuscript.

Study concept was created by LoCasale, Datto, and Coyne. Yeomans and Coyne took the lead in data collection, while data interpretation was performed by LoCasale, Datto, Wilson, and Coyne, with assistance from Yeomans. The manuscript was written by LoCasale, Wilson, Coyne, and Datto, with assistance from Yeomans, and revised by LoCasale, Datto, Wilson, and Coyne.

\section{ACKNOWLEDGMENTS}

Medical writing services were provided by Stephanie Leinbach, $\mathrm{PhD}$, and Erica Wehner, RPh, CMPP, Complete Healthcare Communications, Chadds Ford, Pennsylvania, with funding from AstraZeneca Pharmaceuticals. The authors would like to acknowledge the work of the following employees of AstraZeneca who contributed to the study design and implementation: Fred King, Soheil Chavoshi, and Ron Dirienzi. The authors would also like to acknowledge the work of the following Evidera employees: Jun Chen, Chris Sexton, and Christine Thompson, who contributed to the data analysis and interpretation. Lastly, Colleen Valenzuela and Jersino Jean-Mary at United BioSource Corporation contributed to the study implementation and data collection.

\section{REFERENCES}

1. ACOG Committee Opinion No. 587: Effective patient-physician communication. Obstet Gynecol. 2014;123(2 Pt 1):389-93. Available at: http://journals.lww.com/greenjournal/pages/articleviewer. aspx?year $=2014 \&$ issue $=0200$ 0 \&article $=00036$ \&type $=$ abstract . Accessed December 14, 2015.
2. Travaline JM, Ruchinskas R, D’Alonzo GE Jr. Patient-physician communication: why and how. J Am Osteopath Assoc. 2005;105(1):13-18. Available at: http://jaoa.org/article. aspx?articleid=2093086. Accessed December 14, 2015.

3. Zolnierek KB, DiMatteo MR. Physician communication and patient adherence to treatment: a meta-analysis. Med Care. 2009;47(8):826-34. Available at: http://www.ncbi.nlm.nih.gov/pmc/articles/PMC2728700/. Accessed December 14, 2015.

4. Liker HR, Ducrotté P, Malfertheiner P. Unmet medical needs among patients with gastroesophageal reflux disease: a foundation for improving management in primary care. Dig Dis. 2009;27(1):62-67.

5. Loquai C, Schmidtmann I, Beutel M, et al. Quality of life in melanoma patients during adjuvant treatment with pegylated interferon- $\alpha 2 \mathrm{~b}$ : patients' and doctors' views. Eur J Dermatol. 2011;21(6):976-84.

6. Chou R, Fanciullo GJ, Fine PG, et al. Clinical guidelines for the use of chronic opioid therapy in chronic noncancer pain. J Pain. 2009;10(2):113-30 Available at: http://www.ncbi.nlm.nih.gov/pmc/articles/PMC4043401/pdf/ nihms-578614.pdf. Accessed December 14, 2015.

7. Brown RT, Zuelsdorff M, Fleming M. Adverse effects and cognitive function among primary care patients taking opioids for chronic nonmalignant pain. J Opioid Manag. 2006;2(3):137-46.

8. Mahowald ML, Singh JA, Majeski P. Opioid use by patients in an orthopedics spine clinic. Arthritis Rheum. 2005;52(1):312-21. Available at: http://onlinelibrary wiley.com/doi/10.1002/art.20784/pdf. Accessed December 14, 2015.

9. Cook SF, Lanza L, Zhou X, et al. Gastrointestinal side effects in chronic opioid users: results from a population-based survey. Aliment Pharmacol Ther. 2008;27(12):1224-32. Available at: http://onlinelibrary.wiley.com/ doi/10.1111/j.1365-2036.2008.03689.x/pdf. Accessed December 14, 2015.

10. Camilleri M, Drossman DA, Becker G, Webster LR, Davies AN, Mawe GM. Emerging treatments in neurogastroenterology: a multidisciplinary working group consensus statement on opioid-induced constipation. Neurogastroenterol Motil. 2014;26(10):1386-95.

11. Pappagallo M. Incidence, prevalence, and management of opioid bowel dysfunction. Am J Surg. 2001;182(5A Suppl):11S-18S.

12. Bell TJ, Panchal SJ, Miaskowski C, et al. The prevalence, severity, and impact of opioid-induced bowel dysfunction: results of a US and European Patient Survey (PROBE 1). Pain Med. 2009;10(1):35-42.

13. Bell T, Annunziata K, Leslie JB. Opioid-induced constipation negatively impacts pain management, productivity, and health-related quality of life: findings from the National Health and Wellness Survey. J Opioid Manag. 2009;5(3):137-44.

14. Holzer P, Ahmedzai SH, Niederle N, et al. Opioid-induced bowel dysfunction in cancer-related pain: causes, consequences, and a novel approach for its management. J Opioid Manag. 2009;5(3):145-51.

15. Kumar L, Barker C, Emmanuel A. Opioid-induced constipation: pathophysiology, clinical consequences, and management. Gastroenterol Res Pract. 2014;2014:141737. Available at: http://www.ncbi.nlm.nih.gov/pmc/articles/ PMC4027019/pdf/GRP2014-141737.pdf. Accessed December 14, 2015.

16. Twycross R, Sykes N, Mihalyo M, Wilcock A. Stimulant laxatives and opioid-induced constipation. J Pain Symptom Manage. 2012;43(2):306-13.

17. Coyne KS, LoCasale RJ, Datto CJ, Sexton CC, Yeomans K, Tack J. Opioidinduced constipation in patients with chronic noncancer pain in the USA, Canada, Germany, and the UK: descriptive analysis of baseline patientreported outcomes and retrospective chart review. Clinicoecon Outcomes Res. 2014;6:269-81. Available at: http://www.ncbi.nlm.nih.gov/pmc/articles/ PMC4041290/pdf/ceor-6-269.pdf. Accessed December 14, 2015.

18. Frank L, Kleinman L, Farup C, Taylor L, Miner P Jr. Psychometric validation of a constipation symptom assessment questionnaire. Scand J Gastroenterol. 1999;34(9):870-77. 
19. Reilly MC, Zbrozek AS, Dukes EM. The validity and reproducibility of a work productivity and activity impairment instrument. Pharmacoeconomics. 1993;4(5):353-65.

20. Rabin R, Oemar M, Oppe M. EQ-5D-3L user guide: basic information on how to use the EQ-5D-3L instrument. Rotterdam: EuroQol Group; 2011.

21. Pleil AM, Coyne KS, Reese PR, et al. The validation of patient-rated global assessments of treatment benefit, satisfaction, and willingness to continue-the BSW. Value Health. 2005;8(Suppl 1):S25-34.

22. Eder L, Thavaneswaran A, Chandran V, Cook R, Gladman DD. Factors explaining the discrepancy between physician and patient global assessment of joint and skin disease activity in psoriatic arthritis patients. Arthritis Care Res (Hoboken). 2015;67(2):264-72.

23. Hudson M, Impens A, Baron M, et al. Discordance between patient and physician assessments of disease severity in systemic sclerosis. J Rheumatol. 2010;37(11):2307-12.

24. von Eisenhart Rothe A, Bielitzer M, Meinertz T, Limbourg T, Ladwig $\mathrm{KH}$, Goette A. Predictors of discordance between physicians' and patients' appraisals of health-related quality of life in atrial fibrillation patients: findings from the Angiotensin II Antagonist in Paroxysmal Atrial Fibrillation Trial. Am Heart J. 2013;166(3):589-96.
25. Castrejón I, Yazici Y, Samuels J, Luta G, Pincus T. Discordance of global estimates by patients and their physicians in usual care of many rheumatic diseases: association with 5 scores on a Multidimensional Health Assessment Questionnaire (MDHAQ) that are not found on the Health Assessment Questionnaire (HAQ). Arthritis Care Res (Hoboken). 2014;66(6):934-42.

26. Takeuchi EE, Keding A, Awad N, et al. Impact of patient-reported outcomes in oncology: a longitudinal analysis of patient-physician communication. J Clin Oncol. 2011;29(21):2910-17. Available at: http://jco.ascopubs.org/ content/29/21/2910.full.pdf+html. Accessed December 14, 2015.

27. Ghosh S, Mitchell R. Impact of inflammatory bowel disease on quality of life: Results of the European Federation of Crohn's and Ulcerative Colitis Associations (EFCCA) patient survey. J Crohns Colitis. 2007;1(1):10-20. Available at: http://ecco-jcc.oxfordjournals.org/content/eccojc/1/1/10.full.pdf. Accessed December 14, 2015.

28. August KJ, Nguyen H, Ngo-Metzger Q, Sorkin DH. Language concordance and patient-physician communication regarding mental health needs. J Am Geriatr Soc. 2011;59(12):2356-62.

29. Crawley JA, Horowicz-Mehler N, Hawryluk EA, King F. Impact of opioid-induced constipation: a multinational cross-sectional survey of patients and health care providers [Abstract PGI21]. Value Health. 2013;16(3):A215. Available at: http://www.valueinhealthjournal.com/article/S10983015(13)01162-5/pdf. Accessed December 14, 2015. 